\title{
Analysis of the Ways to Create Intrinsic Safe Enterprises
}

\author{
Yajing $\mathrm{Bi}^{1}$,Yi He${ }^{1}$, Qi Wang ${ }^{2}$ \\ 1. China Academy of Safety Science and Technology,Beijing,China \\ 2. State Grid YangZhou power supply company \\ 13810748726@163.com,25944859@qq.com,23488330@qq.com
}

\begin{abstract}
Intrinsic safety is the inherent safety feature of an integrated enterprise system. The realization of the intrinsic safety of an enterprise depends not only on improving the safety level of "equipment" and "environment" through science and technology, but also on improving the safety quality of the "personnel" and enhancing the safety consciousness of the "personnel". The enterprise should build a sound safety production risk management system, comprehensively analyze the characteristics of intrinsically safe enterprises, analyze the main factors affecting the company's safety production from different perspectives such as "people, equipment, environment and management”, and propose suggestions for creating safe companies.
\end{abstract}

\section{Keywords- intrinsically safe, enterprise, creation}

\section{THE ESSENCE OF INTRINSIC SAFETY}

The concept of intrinsic safety originated from the world aerospace technology industry in the 1950s. It mainly means that electrical equipment has the safety performance to control the release of energy and avoid igniting explosive substances. The concept of intrinsic safety was explicitly proposed in domestic academic studies in the 1990s. Since then, research on intrinsic safety theory has mushroomed, but its scope and connotations are different.

"Intrinsic safety" is an attribute of an enterprise' integrated system. It is an inherent safety feature of the system. It is based on the characteristics and laws of the things themselves. It eliminates or reduces the number of dangerous substances or dangerous operations in the process or equipment, so as to avoid danger rather than control danger; "Intrinsically safe" is the process by which the system achieves or possesses the property of "intrinsically safe"; "Intrinsic safety management" is a general term for a series of actions, measures, or modes of management, regulation, or control that are implemented as close to intrinsically safe as possible.Among them, the equipment is the basis of intrinsic safety, the personnel is the leading of intrinsic safety, the environment is the support of the intrinsic safety, and the management is the guarantee of the intrinsic safety;

"Intrinsically Safe Enterprise" refers to an enterprise that implement "Intrinsic Safety Management" through the "Intrinsically Safe" construction process, thereby making the enterprise "Intrinsically Safe".

\section{INTRINSICALLY SAFE ENTERPRISE FEATURES}

A. Use intrinsic safety principles to increase the safety of production processes, equipment and the environment

Intrinsically safe companies use intrinsic safety principles (such as minimize, replace, weaken, limit errors, and simplification, etc.) to design production processes and select equipment so that production processes, equipment, and the environment are highly safe. The safety of the production system depends mainly on the system itself rather than relying on additional safety devices and measures.

\section{B. To improve the system's synergy by "human-machine} complementarity and human-machine approximation"

In the production process, no major accidents occur due to human unsafe behavior or unsafe conditions of the objects, a system of "human-machine complementarity and humanmachine approximation" is formed. Equipment which plays an important role in safety production is equipped with monitoring, alarm, interlocks, and safety devices make it highly reliable.

\section{Implement internal management of the company by "all staff, the whole process, all-round system method"}

Intrinsically safe enterprises introduce the management method of occupational health and safety management system into internal safety management, and strengthen the internal safety management of enterprises by normative, standard and systematic requirements. Starting from the integrity of the system, we will coordinate and control the subsystems of "human, machine, environment, and management" which involved in safety production work, and emphasize the safety management of all staff, the whole process, and all-round system.

\section{Continuously improve the level of intrinsic safety with}

\section{"continuous improvement"}

Each time the intrinsic safety management system undergoes a PDCA cycle, some safety management problems will be solved and the safety management level will rise to a new height. Then a higher safety goal will be proposed and a new round of PDCA cycles will be started. With the continuous operation of the PDCA cycle, the 
enterprise's intrinsic safety management level will be continuously improved.

\section{THE FACTORS THAT AFFECT THE SAFE PRODUCTION OF ENTERPRISES}

\section{A. Human factors}

(1) Psychological factors

From the psychological factors of people, it is divided into intentional and unintentional. Intentional behavior is to achieve some kind of inappropriate need, and willing to take risks in order to obtain a certain benefit, and make a wrong choice between avoiding risk and gaining benefits. Involuntary behavior refers to unconscious or unintentional behavior. Once it is recognized, it will be promptly corrected. Whether it is intentional behavior or unintentional behavior, it may damage normal production and endanger production safety.

\section{(2) Physiological factors}

The high work intensity, long working hours, heavy manual labor load, and poor working environment during operation all cause the operators to be in a sub-healthy state, making them unable to perform their operations, resulting in operational errors or even accidents.

\section{(3) Skill factors}

There are different levels of employees from management staff to grassroots team members, and there are also employees of different types of work in the company. Some workers have low skills and lack of safety knowledge. They only rely on their poor knowledge and risk management. Some workers have the necessary safety knowledge, but they have poor safety skills, lack of on-site experience, and have poor emergency response capability to emergencies.

\section{B. Equipment factors}

\section{(1) Quality defects}

The quality of equipment and facilities cannot meet the requirements for safe operation. It is prone to accidents during use. Quality defects include design defects and manufacturing defects, as well as the lack of attention to equipment supervision and factory testing.

\section{(2) Inappropriate use, repair and maintenance methods}

The operators are unfamiliar with the operation technique, and use the equipment in a supercharged manner, or they do not maintain the equipment in time during use, resulting in low sensitivity and reliability of the mechanical equipment, and the equipment is in an unsafe state.

(3) Technical equipment is backward

The degree of mechanization, automation, and informationization of technical equipment is not high enough. Severely flawed, unqualified safety tools, construction equipment, safety protection equipment, instrumentation, etc. may cause personal accidents, power grid accidents, equipment accidents or obstacles.

\section{Environmental factors}

The production system is in an open environment. "Opening not only means that the system and the environment exchange material, energy and information, accept input and vibration from the environment, provide output to the environment, but also has the meaning of initiative and evolution." Narrow work spaces, crossoperations, and inclement weather can all lead to accidents.

\section{Management factors}

\section{(1) Organization and management factors}

Some enterprises have a serious shortage of work safety supervision; the safety management system is out of the actual work of safety production, and it is lack of pertinence and operability; there are many labor management mistakes, illegal command, no rules to follow, and cross-operation management confusion.

\section{(2) Site management factors}

In the on-site operation process, some operators did not handle the relationship between safety, quality and progress. The operation process was arbitrarily, the safety hazards were numerous, and the site operations were not standardized. some safety managers were not responsible for safety inspection, and they can not actively find problems and solve problems, resulting in on-site safety inspection workflow in the form.

\section{WAYS TO CREATE AN INTRINSICALLY SAFE POWER COMPANY}

\section{A. Establishing All-Round Safety Concepts to Guide Essential Security Construction}

Through education, make all employees establishe a safety concept of "life is supreme and safety first" and a safety vision of "creating an intrinsically safe enterprise", and this idea and vision are deeply rooted in thoughts and actions.

The concept of safety is mainly the view of safety first, prevention oriented, safety is productivity, safety is benefit, safety is advanced, risk is minimized, safety management is scientific, and self-protection awareness, and prevent problems before they become aware. Only by establishing a safety concept that meets the requirements of intrinsically safe construction can we have the safety activities that are required for the safe production of enterprises, and thus achieve intrinsic safety.

\section{B. Constructing Intrinsically Safe Management System to Realize Intrinsic Safety}

(1) The intrinsic safety of personnel

In the production process, the main measures to reduce people's unsafe behavior are to strengthen the construction of management personnel, improve staff safety skills, and strengthen employee safety responsibilities and standardize operating awareness. The training of management personnel, professional ability, decision-making ability and management ability should be continuously increased. 
(1) Implementation of the full-entry access system. All employees should hold certificates, which prvoid their education on safety, so that everyone can establish a "safety first" ideology, make leaders and staffs improve safety production responsibility and consciousness.

(2) Strengthen employee safety technology training. To enable the majority of employees to master the scientific knowledge of safety technology, to understand the objective law of accidents and development, and to improve the level of safety technology, so that employees can work in accordance with the rules and formal operations at the scene.

(3) Implement standardized operations. Standardizes people's behavior according to scientific operating standards. Operational standardization organically integrates management, technology, and operations, so that management is structured, operations have procedures, and actions have standards. This limits and constrains human errors and eliminates human Unsafe behavior.

(2) The intrinsic safety of the equipment

The intrinsic safety of enterprise equipment requires that machinery and equipment have high reliability, good safety features, complete safety protection systems, and safety protection systems. Even if human errors or equipment accidents occur, they can ensure the safety of people and equipment.

(1) Strengthen equipment management and eliminate defects in the equipment itself. Vigorously develop and promote the use of advanced safety production technologies, constantly improve the safety protection devices, emergency stop and brake devices, and remote alarm devices of machinery and equipment. Strengthen the safety inspection of mechanical and electrical equipment and facilities. Make sure of the maintenance of large units, large equipment and the management of safety components. Strictly monitor the quality of newly purchased equipment safety protection equipment and improve the intrinsic safety level of equipment.

(2) Continuously improve the mechanization and intelligence of the equipment. It is necessary to scientifically and reasonably formulate and implement an intrinsically safe equipment plan based on actual needs, and strive to realize the intelligentization, modernization, and mechanization of safety production equipment so as to increase the intrinsic safety of the machinery.

(3) Increase the pace of information and digital construction. Through information construction, optimizing business processes, advancing scientific corporate management, establishing a basic database for enterprise production and operation and safety management, achieving resource sharing, information integration, providing decision-making basis for safety management, improving safety management efficiency, and enhancing the company's core competitiveness.

(3) The intrinsic safety of the environment
The intrinsic safety of the operating environment means that the production site should ensure the safety of operators, take people as the center, adopt safety technical measures as far as possible, create better production, living environment for the employees, ensure the employees are happy and enjoy a pleasant job, eliminating insecurity and worries, effectively curbing the unsafe behavior of operators in the operating environment and controlling the occurrence of accidents.

In the production practice, based on the actual needs of environmental safety and management to prevent and control accidents. Production accidents can be prevented and controlled in advance to achieve the essential safety of the operating environment.

(4) The intrinsic safety of the management

Modern enterprise security management requires advanced scientific management methods. Intrinsically safe management is one of the key measures to prevent accidents. Its goal is to enhance the company's intrinsic safety level, so that it can have the ability to prevent accidents from the root and in essence.

\section{(1) Quantitative management}

Intrinsic safety management analyzes and quantifies the intrinsic safety capabilities of all types of elements, and provides quantified values for measuring intrinsic safety. Through continuous improvement, the entire process of safety production is managed quantitatively.

\section{(2)System Management}

Intrinsic safety management implements the "all-round, full-process, and full-production cycle" safety management of the company, which means all the company's safety production related objects are all included in the scope of management.

\section{(3) Continuous management}

The improvement of the intrinsic safety level of an enterprise is not an overnight process, but a process of continuous improvement. If intrinsic safety management cannot be implemented consistently, the intrinsic safety level of the company cannot be continuously improved.

\section{Establishing a Safety Culture and Supporting Essential Security Construction}

Safety culture is the sum of all the characteristics and attitudes that exist in organizations and individuals. The realization of intrinsic security requires the support of a safety culture. The safety culture relies on an atmosphere, a spirit, and a kind of psychological induction to influence and coordinate the relationship between people in safety production, and plays an invisible role in guiding and influencing. Through the construction of a safety culture, the sense of instinctiveness of employees' safety and selfprotection can be enhanced, and the self-safety protection capability under the condition of unsupervised monitoring can be strengthened. A safety culture is like an "invisible hand" that promotes safety management. It integrates "selfdiscipline, self-control, self-regulation, and self-prevention" 
into production behavior. This is conducive to inspiring people's consciousness of safe production, stimulating the vitality and creativity of safety production, forming a positive attitude of safety and responsibility for everyone, sharing safety concepts, consciously standardizing safety behaviors, Change from passive to active to fight for security, change "requiring me to be safe" to "I want to be safe".

\section{CONCLUSION}

Production is a complex system engineering. Many factors affect the safety production of enterprises. The complexity determines that there are high risks in the process of production and supply. These risk factors are the main causes of frequent accidents and can bring a huge loss to enterprises. Pre-judgment and prevention of risk accidents, and how to more economically reduce or eliminate safety production risks, and ensure the stable and safe operation of enterprises are issues that some enterprises urgently need to solve. Establishing a sound safety production risk management system is the fundamental way to solve the above problems.

\section{ACKNOWLEDGMENT}

This work is supported by National Key R\&D Program of China (No.2017YFC0805100, 2016YFC0801305) and basic scientific research project founded by China Academy of Safety Science and Technology(No.2017JBKY07, 2018JBKY04). All funding supports are gratefully acknowledged.

\section{REFERENCES}

[1] Dayan Sun, Xiaotao Zhang, Chenggong Guo, Dongdong Xu, Yajing Bi. Ways to Create Intrinsically Safe Power enterprises [J]. Electric Safety Technology,2018,20(01):5-8.

[2] Yajing Bi, Ziqi Xuan.A Brief Analysis of the Causes and Prevention of Limited Space Operation Accidents in Industry and Trade[C].2017 International Conference of Public Safety Science and Technology, 2017:945-947.

[3] Yajing Bi, Qian Hou, Huajun Zhang.The Enlightenment of Practical Experience of Dust Explosion Protection in Developed Countries to China[C]. 2018 Joint International Advanced Engineering and Technology Research Conference,2018:261-265.

[4] Nan Zhang, Zhaoxi Zou.Probe into the establishmen to fessence safety coal mine[J].Journal of Safety Science and Technology,2011,06:180-183. 